\title{
Harnessing multilayer networks to predict metacommunity responses to global environmental change
}

\author{
Tyler McFadden ${ }^{1}$ and Rodolfo Dirzo ${ }^{1}$ \\ ${ }^{1}$ Stanford University
}

December 23, 2020

\begin{abstract}
Species interact both within and across communities, forming complex networks of biotic interactions and spatial links that underpin ecosystem functioning. However, while recent technological advances permit the analysis of increasingly complex and realistic ecological networks, data availability remains a major challenge. Here, we present a novel approach that uses readily available ecological data to build spatially-structured species interaction networks and predict metacommunity responses to environmental change. Predictive Multilayer Networks (PMNs) model the distributions, interspecific interactions, and spatial connectivity of multiple species across a landscape and quantify network structure and stability. We provide a proof-of-concept using a simulated plant-pollinator community, measure network centrality to identify areas of high functional connectivity, and compare land cover scenarios to predict effects of forest loss and restoration on PMN connectance and robustness. PMNs synthesize network approaches from community and landscape ecology and offer a flexible, predictive approach for examining the spatial dynamics of species interactions.
\end{abstract}

\section{Hosted file}

McFadden\&Dirzo_2020Dec11.pdf available at https://authorea.com/users/384698/articles/ 500240-harnessing-multilayer-networks-to-predict-metacommunity-responses-to-globalenvironmental-change 